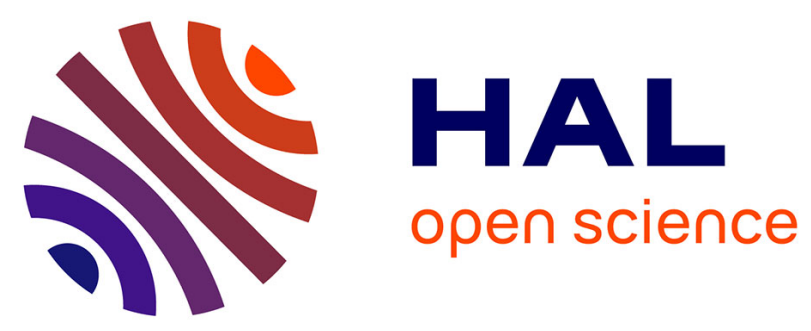

\title{
Modelling of a planar magnetic micropusher for biological cell manipulations.
}

Michaël Dauge, Michaël Gauthier, Emmanuel Piat

\section{To cite this version:}

Michaël Dauge, Michaël Gauthier, Emmanuel Piat. Modelling of a planar magnetic micropusher for biological cell manipulations.. Sensors and Actuators A: Physical , 2007, 138, pp.239-247. hal00162038

\section{HAL Id: hal-00162038 \\ https://hal.science/hal-00162038}

Submitted on 12 Jul 2007

HAL is a multi-disciplinary open access archive for the deposit and dissemination of scientific research documents, whether they are published or not. The documents may come from teaching and research institutions in France or abroad, or from public or private research centers.
L'archive ouverte pluridisciplinaire HAL, est destinée au dépôt et à la diffusion de documents scientifiques de niveau recherche, publiés ou non, émanant des établissements d'enseignement et de recherche français ou étrangers, des laboratoires publics ou privés. 


\title{
Modelling of a Planar Magnetic Micropusher for Biological Cell Manipulations
}

\author{
Michaël Dauge, Michaël Gauthier and Emmanuel Piat \\ Laboratoire d'Automatique de Besançon - CNRS, ENSMM, UFC \\ 24, rue Alain Savary - 25000 Besançon - France \\ Tel: +33 (0) 381402810 - Fax: +33 (0) 381402809 \\ Website: http://www.lab.cnrs.fr, E-mail: michael.gauthier@ens2m.fr
}

\begin{abstract}
The improving of the efficiency and the automation of biological cell technologies is currently of great importance. One way is to build biological micro-factories which are able to perform complete biotechnological processes automatically. This technology requires the development of new automatic cell transport system to feed work stations in microfactories. An original magnetic cell micropusher is described in this paper. The ferromagnetic pusher which is submerged in the biological medium follows the movement of a permanent magnet located in the air. This paper focuses on the modelling of the dynamic behaviour of the micropusher according to the magnet trajectory. The generic model proposed is able to determine pusher trajectory according to the micropusher magnetic properties and the permanent magnet shape and properties. This simulation tool will permit to optimize and to study cell trajectory control in further works.
\end{abstract}

Area - Micromechanics.

Key words - Micromanipulation, magnetic actuation, modelling, scale effect, biological cell.

\section{INTRODUCTION}

Although the In-Vitro Fertilization (IVF) process is usually used by physicians, the success rate remains particularly low. Thus, the improving of this biological process with new biological technologies and new microtechnologies is of great importance. Some microtechnology research groups are working towards a high efficiency IVF process [1-7]. The IVF process whose goal is to fertilize oocytes consists of several manually or teleoperated manipulation steps and requires a high pratical know-how. The major part of the full process time is needed to prepare the oocytes before the technical operations. The typical size of a human oocyte is around $150 \mu \mathrm{m}$ diameter.

Since all steps of the current IVF process are manually made or teleoperated, the design of an automatic IVF microfactory is a solution to improve the ergonomics of current processes and to guarantee a better repeatability of manipulations and injections. In this way, the efficiency of IVF (around $30 \%$ of pregnancies at the present time in Europe) should improve.

Furthermore automated manipulations and oocyte treatments will enable other biotechnologies like embryonic stem-cell research for therapeutic cloning to develop. Presently, the objective of the therapeutic cloning is to build organs from one stem cell. These processes require the culture of a large number of oocytes. In this way, an oocyte treatment microfactory could contribute to therapeutic cloning.

Our microfactory project is presented in figure 1. This bio-microfactory will contain modular microrobots and a cells transport system to move cells inside the microfactory. Each modular microrobot will have a specific function: Oocyte cumulus removal, oocytes properties measurements, fertilization. 


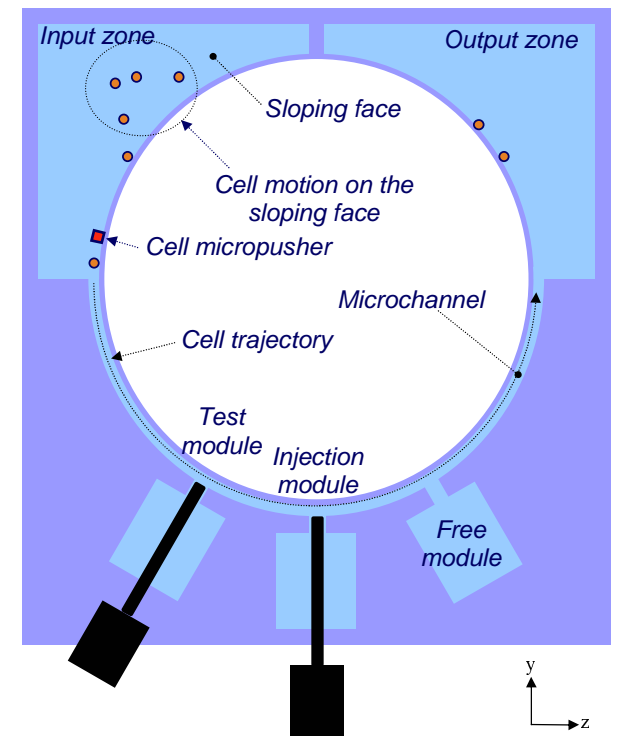

Fig. 1. In Vitro Fertilization Microfactory Concept

This work is done in collaboration with the physician: Prof. C. Roux of the Genetic and Reproduction research team of the University of Franche-Comté, France.

The oocytes are placed in the input zone after cumulus removal (see figure 1). Oocytes go down on the sloping face and stand motionless against the wall. The transfer of oocytes from the input zone to the output area through the processing modules is achieved by a cell micropusher. Some standard modules (test or treatment modules) are placed by the physicians along the cell trajectory. At the end of the process, injected oocytes are placed in the output area where physicians can catch them.

The conventional glass micropipette is a competitive tool for the manipulation of oocytes. However, complex trajectories, complex shunting, or manipulation of an oocyte placed behind another oocyte cannot be performed with conventional micropipettes. Consequently, we propose a new technology allowing complex oocyte trajectories. This article presents a two degree-of-freedom (DOF) magnetic cell transport system for automated IVF micromanipulation. Previous works on cell magnetic micropushers concerned a one DOF configuration which was not suitable with current inverted microscope used in biological micromanipulations [7], [8].

The following section deals with the description of the oocyte transport system and open-loop oocyte micromanipulations. The third section focuses on the modelling of the pusher magnetic behaviour and dynamic behaviour. Experimental trajectories and comparisons with the models are described in the last section.

\section{Oocyte Transport System}

The aim of our oocyte transport system is to enable cell transport in a channel through the different work stations. The channel volume is filled with a biological medium ( see figure 1). To move our actuator, we chose to use magnetic energy which is a suitable solution for micro-actuation [9-18]. Moreover static magnetic field has no effect on living cells unlike laser trapping systems [19].

The original single cell transport system consists of a wireless micropusher which pushes a single cell 


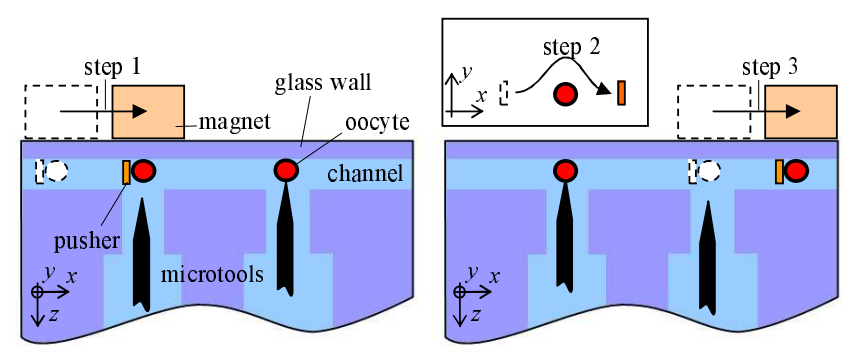

Fig. 2. Example of Cells Treatment Micromanipulation Strategy: Micropusher places an oocyte in front of the first tool (step1). When the second tool operation is finished, the micropusher goes over the first oocyte (step 2) and evacuates the second oocyte (step 3).

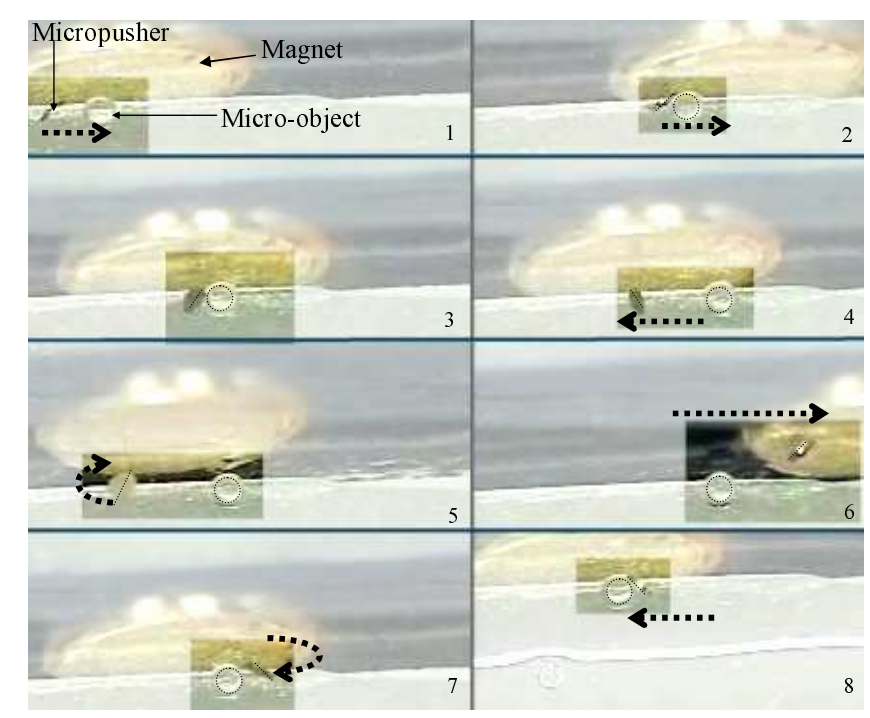

Fig. 3. Example of Automatic Open-loop Micromanipulation: The micropusher comes near the micro-object (1), pushes it (2), stops its movement (3), goes back (4) and goes over the micro-object (5) to goes on the other side him (6), comes near the micro-object (7) and pushes it (8) in the other direction

without contact with the external medium. When the cells manipulated are oocytes, the micropusher is a ferromagnetic particle $\left(240 \times 240 \times 25 \mu \mathrm{m}^{3}\right)$ made in electroplated nickel [20] and actuated with an external magnetic field. In our case, the external magnetic field is induced by a cylindrical magnet $(\mathrm{NdFeB}, \phi 1.6 \mathrm{~mm}, \mathrm{~h}=0.74 \mathrm{~mm})$ placed on the other side of a vertical wall. Contrary to microfluidic transfer [21], our proposed micropusher is able to push each cell individually. In the future, micropushers will be encapsulated because nickel is embryotoxic. The micropusher and cell positions are measured through an optical microscope and a CCD camera. The displacements are made by two motorized micro-translation stages $\left(\mathrm{PI}{ }^{\circledR}, \mathrm{M}-111.1\right)$ controlled by a computer.

Figure 2 describes an example of some micropusher trajectories used to position oocytes. It's a linear view of the in vitro fertilization microfactory presented in figure 1 . The first degree of freedom (DOF) along $x$ axis is used to push the micro-object (step 1 or 3 ). The second DOF along $y$ axis is used to go over the micro-object (step 2). Micropusher placed against the wall is used to position oocytes in front of tools like injector, aspiration pipe, sensor, etc.

To test our system and its feasibility, we did some preliminary automatic open loop micromanipulations. Figure 3 presents a pushing operation on a micro-object (200 $\mu \mathrm{m}$ diameter sphere). This micromanipulation example proves the feasibility of our concept. 


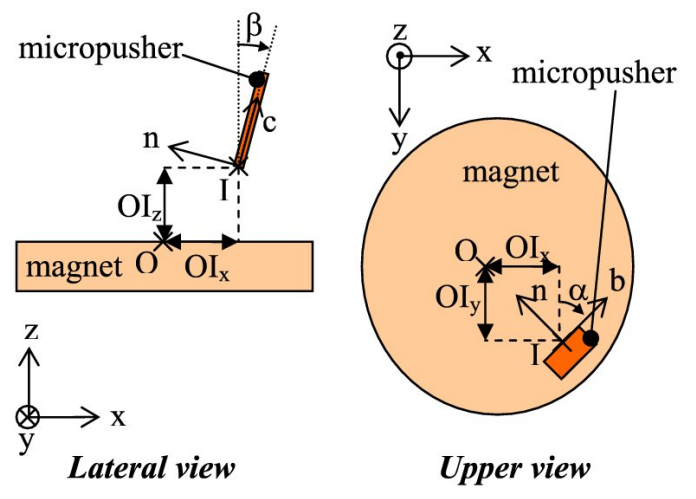

Fig. 4. Magnet Micropusher Position and Orientation

To optimize this principle, a complete modelling of the link between the magnet trajectory and micropusher trajectory is needed. Moreover to perform cell displacements, the micropusher trajectory must be controlled and the study of the automatic control needs a complete dynamic model too. The behaviour modelling is presented in the following.

\section{Behaviour Modelling}

The modelling aims are to propose a complete model of the dynamic behaviour of the micropusher in function of the magnetic source movement where parameters are the shape of the micropusher, and the shape and the nature (permanent magnet, micro-coils) of the magnetic source.

Position between micropusher and magnet, presented in figure 4, is defined by two points: Center $O$ of the magnet top plane and contact point $I$ between the micropusher and the glass wall. The position of the micropusher in an inertial reference frame $R_{m}:\left(O_{m}, \vec{x}, \vec{y}, \vec{z}\right)$ is defined by $x_{m}, y_{m}$, and $z_{m}$. Relative positions between the point $O$ and the point $I$ are defined as $O I_{x}, O I_{y}$ and $O I_{z}$. Micropusher orientation is defined by two angles: $\alpha$ and $\beta$ defined in figure 4. Angle $\alpha$ enables movements like step 2 in figure 2. Angle $\beta$ is useful for accurate positioning [7]. The micropusher is modelled by a plane surface $S$, with a normal $\vec{n}$ and a thickness $e$. Micro-pusher reference frame is defined by vectors: $\vec{n}, \vec{b}$ and $\vec{c}$ described in figure 4 .

The model describes the behaviour of a ferromagnetic particle (whatever its plane shape is) into a magnetic field (whatever its source is). Consequently the model outputs are the position and the orientation of the micropusher, the inputs are the position of the magnetic source and the parameters are magnetic micropusher properties and the magnetic field $\overrightarrow{B_{0}}$ induced by the permanent magnet.

The model is composed of several blocks (see figure 5) where each block represents one calculation step. First the finite element model "Flux3D ${ }^{\complement}$ " is used to obtain the magnetic field $\overrightarrow{B_{0}}$ according to the magnetic source shape and properties. The second step consists of the calculation of the magnetization $\vec{M}$ of the micropusher. Magnetic torque $\overrightarrow{\Gamma_{m, I}}$ in point $I$ and magnetic force $\overrightarrow{F_{m}}$ applied by the magnet on the micropusher are determined in function of the relative position $\overrightarrow{O I}$ between the micropusher and the magnet. These first steps are performed off-line and efforts $\left(\overrightarrow{F_{m}}, \overrightarrow{\Gamma_{m, I}}\right)$ are stored in a large numerical matrix.

The last step is the determination of the micropusher position $\left(x_{m}, y_{m}\right.$ and $\left.z_{m}\right)$ and orientation $(\alpha$ and $\beta$ ) behaviour using the efforts stored. Each block principle is detailed in the following. 


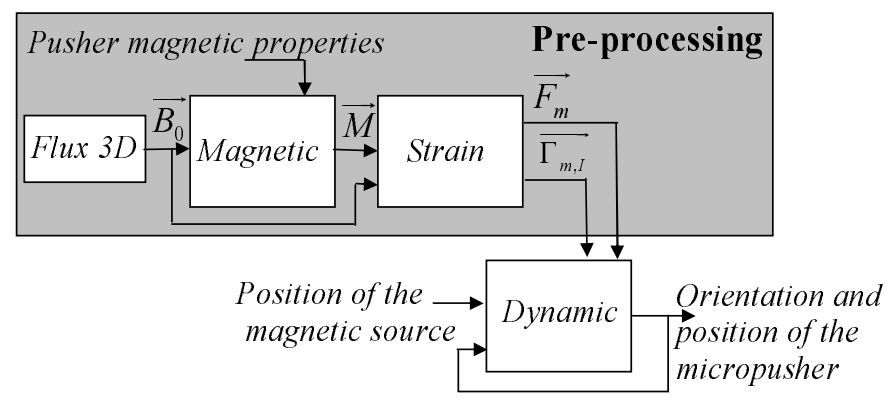

Fig. 5. Micropusher Behaviour Model Diagram

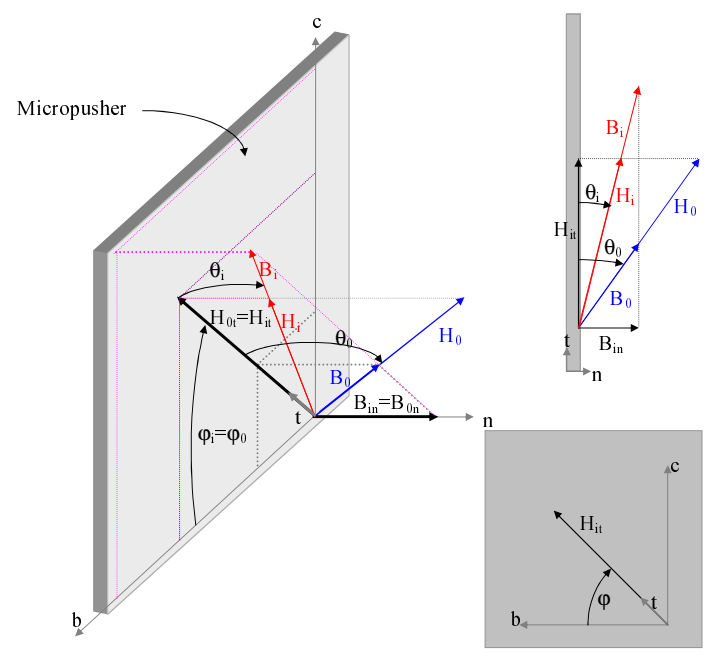

Fig. 6. Magnetic field orientation

\section{A. Magnetic Block}

The aim of this part is to describe the process done by the magnetic block. The block ouput is the micropusher internal magnetization $\vec{M}$ according to the micropusher geometrical characteristic and magnetic field $\overrightarrow{B_{0}}$.

To build this function, we assume two hypotheses:

- the micropusher is a flat surface $S$ : magnetization is constant according to the micropusher thickness,

- edge effects are not taken into account: there is no magnetic leak.

With the magnetostatic Maxwell equations, we can define the continuity relation between $\overrightarrow{B_{0}}$ and the internal micropusher magnetic field $\overrightarrow{B_{i}}$, and the continuity relation between external magnetic excitation $\overrightarrow{H_{0}}$ and internal micropusher magnetic excitation $\overrightarrow{H_{i}}$ (relations $(1)$ and $(2)$ ).

$$
\begin{aligned}
\overrightarrow{B_{0 n}} & =\overrightarrow{B_{i n}} \\
\overrightarrow{H_{0 t}} & =\overrightarrow{H_{i t}}
\end{aligned}
$$

where:

$$
\begin{array}{cc}
\overrightarrow{B_{0}}=\left\|B_{0 n}\right\| \cdot \vec{n}+\left\|B_{0 t}\right\| \cdot \vec{t} & \overrightarrow{B_{i}}=\left\|B_{i n}\right\| \cdot \vec{n}+\left\|B_{i t}\right\| \cdot \vec{t} \\
\overrightarrow{H_{0}}=\left\|H_{0 n}\right\| \cdot \vec{n}+\left\|H_{0 t}\right\| \cdot \vec{t} & \overrightarrow{H_{i}}=\left\|H_{i n}\right\| \cdot \vec{n}+\left\|H_{i t}\right\| \cdot \vec{t}
\end{array}
$$


As described in figure $6, \overrightarrow{B_{0}}$ and $\overrightarrow{H_{0}}$ orientation are defined by $\theta_{0}$ and $\varphi_{0}$, and orientation of both $\overrightarrow{B_{i}}$ and $\overrightarrow{H_{i}}$ by $\theta_{i}$ and $\varphi_{i}$. Our aim is to obtain magnetization $\vec{M} . \vec{M}$ and $\overrightarrow{B_{i}}$ are colinear, so the vector $\vec{M}$ is defined by its module $\|\vec{M}\|$ and the angles $\varphi_{i}$ and $\theta_{i}$. We assume that the magnetization has reached the saturation $M_{\text {sat }}$, thus it gives the following equation:

$$
\begin{aligned}
\|\vec{M}\| & =M_{\text {sat }} \\
& =5.1 \times 10^{5} \text { A.m }{ }^{-1} \quad \text { nickel saturation magnetization }
\end{aligned}
$$

Moreover from relation (2), $\varphi_{i}$ satisfies:

$$
\varphi_{i}=\varphi_{0}
$$

From equations $(1,3,4), \theta_{i}$ satisfies:

$$
B_{0 n}=\tan \left(\theta_{i}\right) \cdot\left(B_{0 t}+\mu_{0} \cdot M_{s a t} \cdot \cos \left(\theta_{i}\right)\right)
$$

The system $(3,4,5)$ defines the vector $\vec{M}$ as a function of the magnetic field $\overrightarrow{B_{0}}$.

\section{B. Strain Block}

To present the mechanical action applied by the magnetic source on the micropusher, a small surface $d S$ of the flat micropusher around the point $P$ is considered. Classical magnetic efforts applied on this element are:

- An elementary surface magnetic force $\overrightarrow{d F_{m}}$ :

$$
\overrightarrow{d F_{m}}=\vec{\nabla}\left(\vec{M} \cdot \overrightarrow{B_{0}}\right) \cdot d S
$$

- An elementary surface magnetic torque $\overrightarrow{d \Gamma_{m}}$ :

$$
\overrightarrow{d \Gamma_{m}}=\vec{M} \wedge \overrightarrow{B_{0}} \cdot d S
$$

The complete magnetic effort applied on the pusher in the point $I$ is thus:

$$
\begin{aligned}
& \overrightarrow{F_{m}}=e \times \int_{S} \overrightarrow{d F_{m}} \\
& \overrightarrow{\Gamma_{m, I}}=e \cdot \int_{S} \overrightarrow{d \Gamma_{m}}+e \cdot \int_{S} \overrightarrow{P I} \wedge \overrightarrow{d F_{m}}
\end{aligned}
$$

where $e$ is the thickness of the flat micropusher.

\section{Dynamic Block}

We assume that the friction force applied from the glass wall to the micropusher $\overrightarrow{F_{f}}$ is applied in point $I$, and consequently that friction torque is null in $I$. The dynamic behaviour is defined by the equilibrium between efforts and dynamic terms in point $I$ :

$$
\begin{aligned}
\overrightarrow{F_{f}}+\overrightarrow{F_{m}} & =m \vec{\Gamma} \\
\overrightarrow{\Gamma_{m, I}} & =J \cdot \vec{\gamma}
\end{aligned}
$$

where $\overrightarrow{F_{f}}$ is the action of the glass wall on the micropusher applied in point $I, m$ the micropusher mass, $\vec{\Gamma}$ its acceleration, $J$ its angular inertia, and $\vec{\gamma}$ the angular acceleration. 
The dynamic behaviour in translation is a function of the magnetic force $\overrightarrow{F_{m}}$ and of friction conditions $\overrightarrow{F_{f}}$. The angular behaviour is only a function of magnetic effects $\overrightarrow{\Gamma_{m, I}}$.

The dynamic behaviour is the superposition of three elementary dynamic behaviours described as:

- The angular behaviour $\alpha$ with axe $(I, \vec{z})$;

- The angular behaviour $\beta$ with axe $(I, \vec{b})$;

- The translation behaviour $\left(x_{m}, y_{m}\right)$.

By computation of the dynamic equations (10), (11) the time constants of these behaviours are determined. These values are presented in table I.

\begin{tabular}{|c|c|}
\hline $\begin{array}{c}\text { Elementary } \\
\text { Dynamic Behaviours }\end{array}$ & Time Constant \\
\hline Rotation $\alpha$ & $45 \times 10^{-6} \mathrm{~s}$ \\
\hline Rotation $\beta$ & $37 \times 10^{-6} \mathrm{~s}$ \\
\hline Translation $\left(x_{m}, y_{m}\right)$ & $930 \times 10^{-6} \mathrm{~s}$ \\
\hline
\end{tabular}

TABLE I

Time Constant of the Three Elementary Dynamic Behaviour

The time constants of the angular behaviour are negligible compared to the translation time constant. Moreover, these time constants are very small compared to the time constant of the controller (around $1 \mathrm{~ms}$ ) or compared to the time constant of the camera $(20 \mathrm{~ms})$ used to measure the micropusher position. Consequently the general dynamic behaviour can be considered as a dynamic behaviour in translation and a quasi-static behaviour in rotation. Both behaviours are detailed in the following sections

1) Angular Behaviour: At each time $t$, we consider that the micro-pusher is reaching its angular static equilibrium. The angular behaviour presented in (11) is consequently reduced as:

$$
\overrightarrow{0}=\overrightarrow{\Gamma_{m, I}}=e \cdot \int_{S} \overrightarrow{d \Gamma_{m}}+e \cdot \int_{S} \overrightarrow{P I} \wedge \overrightarrow{d F_{m}}
$$

The angular position is characterized by the equilibrium between both terms of the magnetic torque $\overrightarrow{\Gamma_{m, I}}$. Consequently, its calculation in function of the relative position $\overrightarrow{O I}$ can be determined in preprocessing. Thus, the dynamic block can be presented as described in figure 7. Angular positions are defined in function of the torque $\overrightarrow{\Gamma_{m, I}}$ and are stored in a large numerical matrix. Translation behaviour is simulated in function of the position of the magnet and the stored magnetic forces. At each time $t$, the angle of the micropusher is calculated by interpolation of the stored angular positions.

2) Translation behaviour: The micropusher translation behaviour (10) is a function of the friction force applied by the glass vertical wall. This force $\overrightarrow{F_{f}}$ can be divided into:

- A normal component $\overrightarrow{F_{f N}} \cdot \vec{z}$ perpendicular to the contact surface which is in equilibrium with the normal magnetic force:

$$
\overrightarrow{F_{f N}} \cdot \vec{z}=-\overrightarrow{F_{m}} \cdot \vec{z}
$$

- A tangential component $\overrightarrow{F_{f T}}$ sum of both components $F_{f T x} \cdot \vec{x}$ and $F_{f T y} \cdot \vec{y}$.

To describe the translation behaviour of the micro-pusher, two cases are studied: 


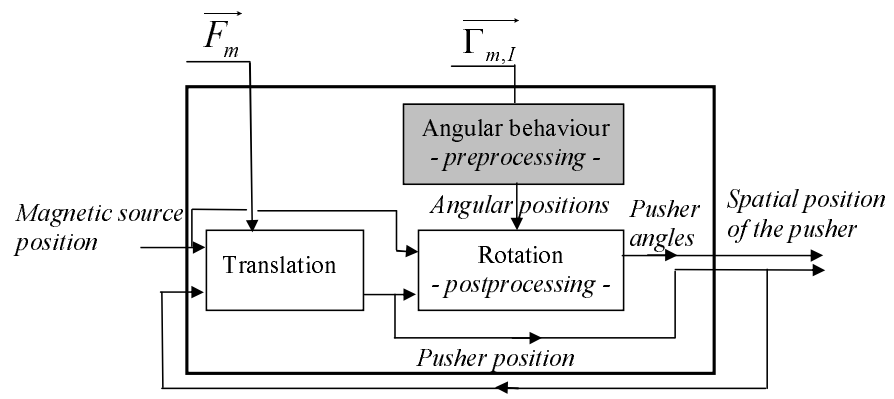

Fig. 7. Dynamic Simulation Model Diagram

- Point $I$ is immobile and the movement of the micropusher is only a rotation;

- Point $I$ moves, and the micropusher is in translation on the vertical glass slide.

When the micropusher contact point $I$ is immobile, the friction tangential component $\overrightarrow{F_{f T}}$ is lower than the product of the static friction coefficient $f_{s}$ and the normal component $\overrightarrow{F_{f N}}$ :

$$
\left\|\overrightarrow{F_{f T}}\right\|<f_{s} \cdot\left\|\overrightarrow{F_{f N}}\right\|
$$

In this case, the tangential component of the friction force and the tangential magnetic force stay in equilibrium:

$$
\left\{\begin{array}{l}
\overrightarrow{F_{f T}} \cdot \vec{x}+\overrightarrow{F_{m}} \cdot \vec{x}=0 \\
\overrightarrow{F_{f T}} \cdot \vec{y}+\overrightarrow{F_{m}} \cdot \vec{y}=0
\end{array}\right.
$$

When the norm of the tangential component reaches the product $f_{s} \cdot\left\|\overrightarrow{F_{f N}}\right\|$, the contact point $I$ moves:

$$
\left\|\overrightarrow{F_{f T}}\right\|=f_{s} \cdot\left\|\overrightarrow{F_{f N}}\right\| \Rightarrow \overrightarrow{V_{I}} \neq 0
$$

where $\vec{V}_{I}$ is the velocity of point $I$.

When the micropusher is in movement with a velocity $\vec{V}_{I}$, the modulus of the friction tangential force is the product of the dynamic friction coefficient $f_{d}\left(f_{d} \leq f_{s}\right)$ and the normal component $\left\|\overrightarrow{F_{f N}}\right\|$ :

$$
\overrightarrow{F_{f T}}=-f_{d} \times\left\|\overrightarrow{F_{f N}}\right\| \cdot \overrightarrow{n_{v}}
$$

where $\overrightarrow{n_{v}}$ is the unit vector of the velocity $\vec{V}_{I}$. 


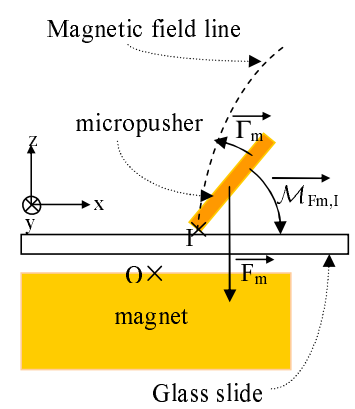

Fig. 8. Strains applied by the magnet on the micropusher

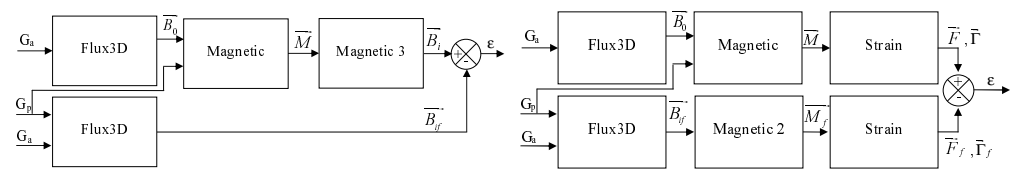

(a) Magnetization comparison be- (b) Forces comparison between our tween our model and Flux3D re- model and Flux3D result sult

Fig. 9. Model and Flux3D comparisons principle

The translation dynamic behaviour of the micropusher is defined by the projection of the equation (10) in the wall plane $(\vec{x}, \vec{y})$, thus:

$$
\begin{aligned}
& \left\{\begin{array}{l}
\overrightarrow{F_{m}} \cdot \vec{x}+\overrightarrow{F_{f T}} \cdot \vec{x}=m \ddot{x}_{m} \\
\overrightarrow{F_{m}} \cdot \vec{y}+\overrightarrow{F_{f T}} \cdot \vec{y}=m \ddot{y}_{m}
\end{array}\right. \\
& \left\{\begin{array}{c}
\overrightarrow{F_{m}} \cdot \vec{x}-f_{d} \cdot\left\|\overrightarrow{F_{f N}}\right\| \cdot \overrightarrow{n_{v}} \cdot \vec{x}=m \ddot{x}_{m} \\
\overrightarrow{F_{m}} \cdot \vec{y}-f_{d} \cdot\left\|\overrightarrow{F_{f N}}\right\| \cdot \overrightarrow{n_{v}} \cdot \vec{y}=m \ddot{y}_{m}
\end{array}\right. \\
& \left\{\begin{array}{c}
x_{m}=\frac{1}{m} \iint\left(\overrightarrow{F_{m}} \cdot \vec{x}-f_{d} \cdot\left\|\overrightarrow{F_{f N}}\right\| \cdot \overrightarrow{n_{v}} \cdot \vec{x}\right) \cdot d^{2} t \\
\quad+\dot{x}_{m}(0) t+x_{m}(0) \\
y_{m}=\frac{1}{m} \iint\left(\overrightarrow{F_{m}} \cdot \vec{y}-f_{d} \cdot\left\|\overrightarrow{F_{f N}}\right\| \cdot \overrightarrow{n_{v}} \cdot \vec{y}\right) \cdot d^{2} t \\
\quad+\dot{y}_{m}(0) t+y_{m}(0)
\end{array}\right.
\end{aligned}
$$

The equations (15) and (20) define respectively the behaviour of the micro-pusher when point $I$ is immobile and when it is in movement. The equation (16) defines the transition condition.

Moreover when $f_{d} \neq f_{s}$, the stick-slip motion appears as presented in [8]. When the magnet moves, the micro-pusher stays first immobile until the magnetic tangential force reaches the static friction condition (equation (16)). Thus, the micro-pusher is moving in function of the dynamic behaviour (equation (20)), and a large acceleration is applied to the micro-pusher until it reaches a new static equilibrium position $\left(\overrightarrow{V_{I}}=\overrightarrow{0}\right)$.

This phenomenon increases the difficulties to control the final position of the micro-pusher or the pushed object position. Some original control strategies are proposed in [7] to overcome stick-slip. 


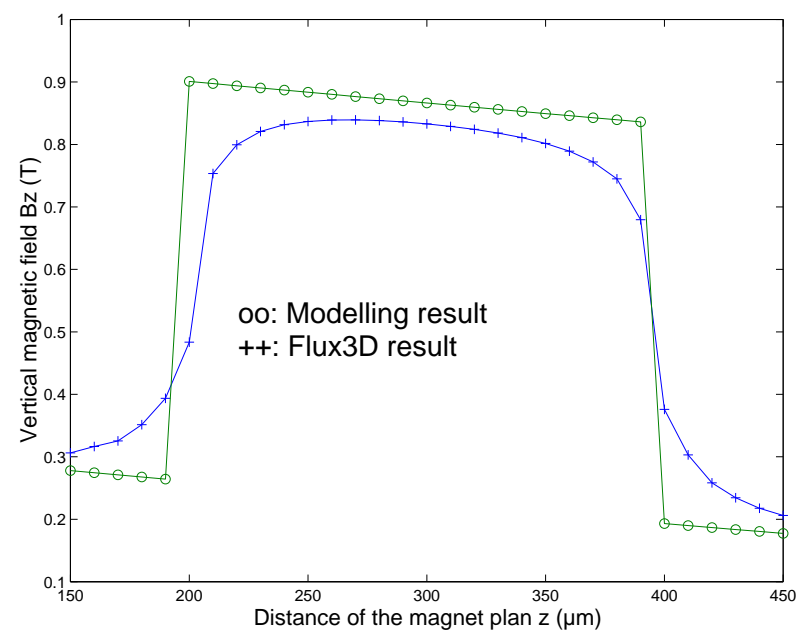

Fig. 10. Model and Flux3D results comparison

\section{ExPERIMENTAL AND SIMULATiOn RESUlts}

\section{A. Magnetic results comparison}

Firstly we have compared the magnetic field with the presence of the micropusher given by our model and by Flux3D model. Results are presented in figure 10 which represents the magnetic field $\overrightarrow{B_{z}}$ (projection of $\overrightarrow{B_{o}}$ into $z$ axis) given by Flux3D and our model magnetic model at point $P$ as a function of the distance $O P_{z}$ between the point $P$ and the magnet surface. The simulation conditions are the following:

- micropusher is placed at the magnet center $O I_{x}=0, O I_{y}=0, O I_{z}=200 \mu \mathrm{m}$;

- consequently the micropusher edges are placed at $O P_{z}=200 \mu \mathrm{m}$ and $O P_{z}=400 \mu \mathrm{m}$.

The consequences of the hypotheses made (no magnetic leak) are visible on the edges of this micropusher, but their influences on forces computation is under $10 \%$ as presented in the next section.

\section{B. Force results comparison}

To validate force calculation and to observe the influence of magnetization errors on forces calculation, force $\overrightarrow{F_{m}}$ was computed with magnetization calculated by Flux3D and our model. Experimental conditions are the following:

- micropusher is placed up to the magnet center $\left(O I_{x}=O I_{y}=0\right.$ and $\left.200 \mu m<O I_{z}<1600 \mu m\right)$,

- micropusher is placed in $\mathrm{xOz}$ plane

The figure 11 presents a comparison between both calculated forces and experimental forces measured with a microbalance, according to the distance $O I_{z} \cdot \overrightarrow{F_{z}}$ is nearly the same with Flux3D and our model.

\section{Angular Behaviour}

The first experimental validation deals with the equilibrium angle of the micropusher (angle $\beta$ in figure 4 ) versus the micropusher size and the relative position $\overrightarrow{O I}$. These tests enable to compare computed and measured values. The calculated angles $\alpha$ and $\beta$ are determined by the numerical resolution of equilibrium equation (12). 


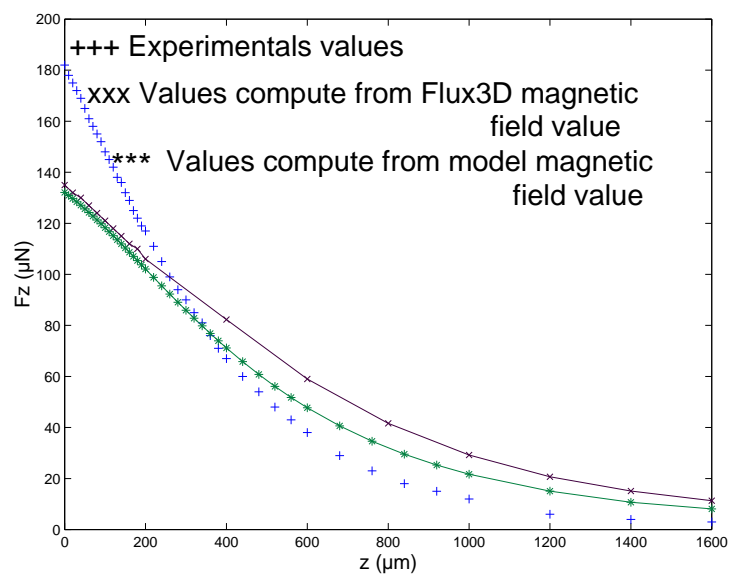

Fig. 11. Comparison between $F_{z}$ measurement and simulations

As shown by equation (12), the angular position is done by the equilibrium of two physical phenomena: The magnetic torques $\overrightarrow{d \Gamma_{m}}$ and the torques in $I$ induced by the magnetic force $\overrightarrow{d F_{m}}$ :

$$
\int_{S} \overrightarrow{d \Gamma_{m}}=-\int_{S} \overrightarrow{P I} \wedge \overrightarrow{d F_{m}}
$$

These two strains induce two different micropusher behaviours:

The magnetic torques $\overrightarrow{d \Gamma_{m}}$ consequence are the alignment of the micropusher on the external magnetic field $\overrightarrow{B_{0}}$ (see equation 7 ). On the contrary, the torque induced by the magnetic force $\overrightarrow{P I} \wedge \overrightarrow{d F_{m}}$ makes the micropusher lie flat on the plane.

In fact the first term is function of $l^{3}$ and the second is function of $l^{4}$ where $l$ is the micropusher characteristic size. Consequently, the smaller the micropusher, the greater is the magnetic torque $\overrightarrow{d \Gamma_{m}}$ compared to the magnetic force torque $\overrightarrow{P I} \wedge \overrightarrow{d F_{m}}$. Physically, the smaller the micropusher, the closer is its orientation to the magnetic field line (see figure IV-C).

Simulations and experimental measurements are presented in figure IV-C for the following case: $-500 \mu m<O I_{x}<+500 \mu m ; \mathrm{OI}_{y}=0 ; \mathrm{OI}_{z}=200 \mu \mathrm{m}$.

The micropusher is not aligned on the magnetic field $\overrightarrow{B_{0}}$. Actually, magnetic torque is not prominent compared to the magnetic force torque. Conversly, the micropusher is also not lying down on the glass slide because magnetic force torque is not prominent compared to the magnetic torque. Both phenomena have the same value order and the micropusher orientation is defined between these two extremum positions. Experimental measurements and our model are similar.

\section{Dynamic Comparison}

This section presents the comparison between the computed trajectories and experimental measurements. The simulation of the pusher trajectory is based on the magnetic force calculation (8) computed for each relative position $\overrightarrow{O I}$ in preprocessing. Simulated trajectory is obtained by resolution of the equations (15), (16) and (20) in Matlab Simulink. The experimental position of the micro-pusher is measured by a CCD Camera and a visual treatment.

The example presented in figure IV-D is obtained with a square trajectory of the magnet. A good correlation between both results is observed, and trajectories are relatively near. 


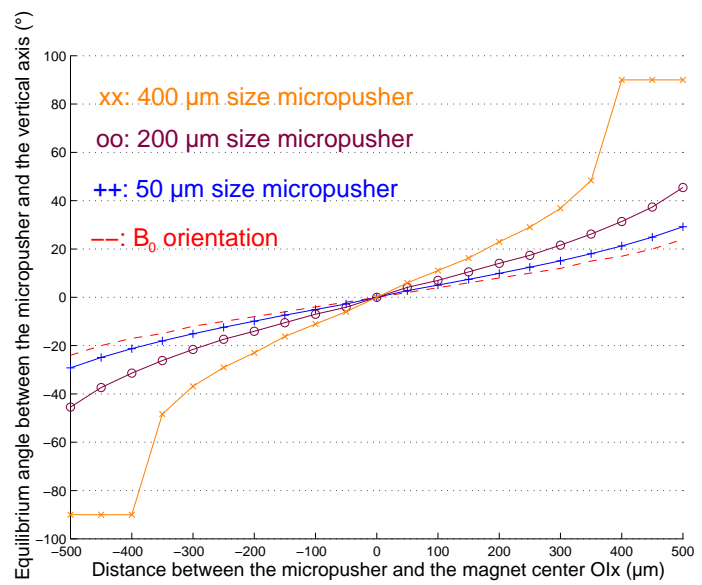

Fig. 12. Micropusher height influence on the micropusher orientation $\beta$

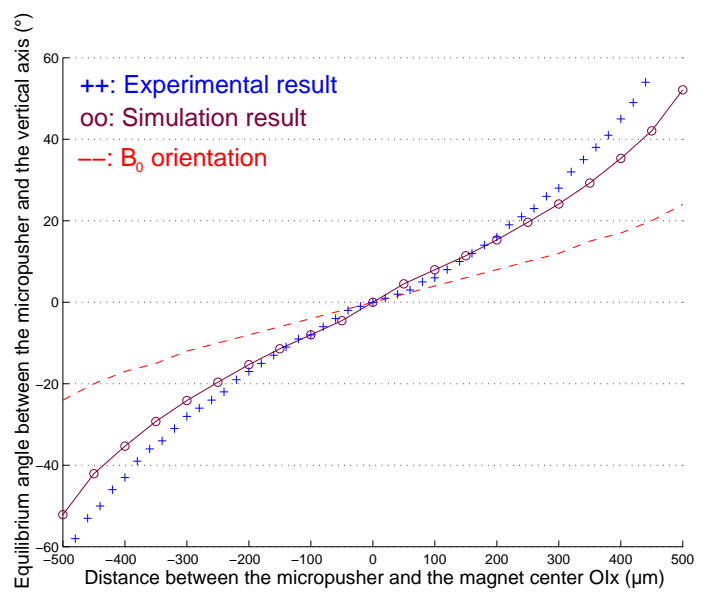

Fig. 13. Comparison Between Experimental and Simulated Micropusher Angles $\beta$

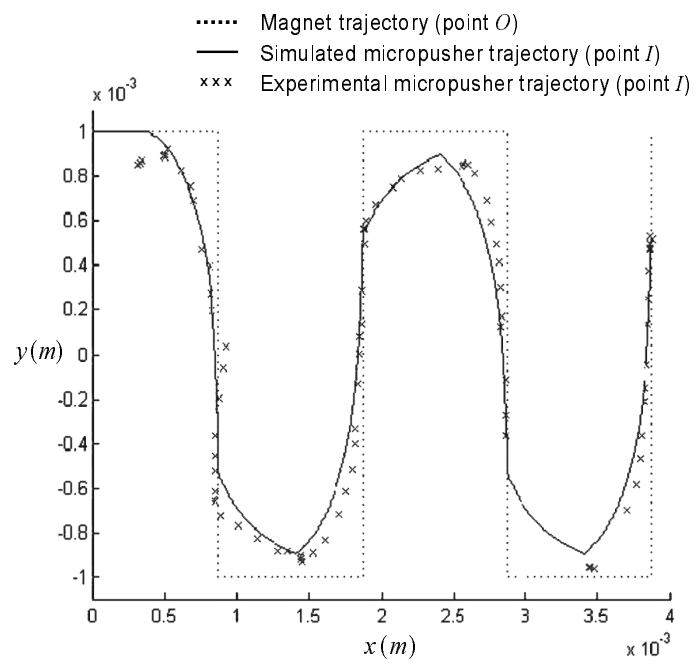

Fig. 14. Micro-pusher and Magnet Trajectories: Comparison Between Computed Values and Experimental Measurements. 


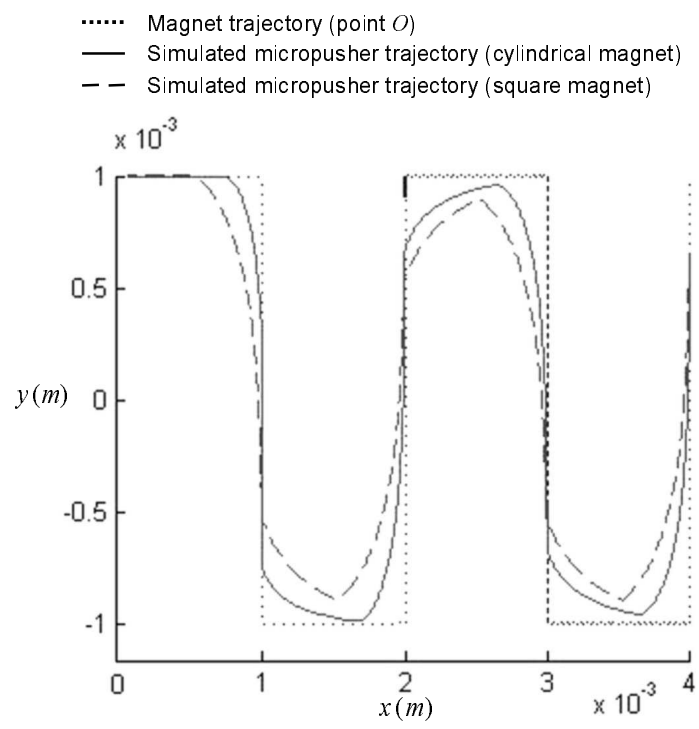

Fig. 15. Micro-pusher and Magnet Trajectories: Influence of the Magnet Shape on the Micro-pusher Trajectory.

Moreover, the model permits to compute trajectories in function of the magnetic source shape. We present the differences observed between two permanent magnets:

- A cylindrical magnet $1.6 \mathrm{~mm}$ in diameter, $0.74 \mathrm{~mm}$ in thickness and axis $z$;

- A square magnet $\left(1.6 \times 1.6 \times 0.74 \mathrm{~mm}^{3}\right)$.

Both magnets have a remanent magnetic field oriented along the $z$ axis. Both trajectories are presented in figure IV-D.

The second geometry induces a modification of the magnetic field $\overrightarrow{B_{0}}$ simulated by the software Flux 3D. This modification induces a new dynamic behaviour and a modification of the micro-pusher trajectory.

The model build is designed to be generic and is able to determine the dynamic behaviour of the micropusher in function of the magnet movement for different magnet shapes, and micropusher shapes and properties. Moreover, as the model is only based on the magnet field induced by a magnetic source it can be generalized to micro-coils.

\section{Discussion And Conclusion}

We propose in this paper a new and original structure of IVF process. The displacement of the cells in the biological microfactory is induced by a micropusher which follows the movement of a permanent magnet. This method is more flexible than the current technologies which are using micropipettes. Some other methods are studied around the world to manipulate cells like laser trapping [1] and dielectrophoresis micromanipulation [2]. Both technologies can induce irreversible damages to cells. The pushing action is more safety and is usually used with micropipettes. However, our nickel micropusher remains embryo toxic and cannot be used without biocompatible coating. This encapsulation will not disturb the magnetic behavior of the nickel micropusher but will modify the friction conditions of the micropusher, and consequently the trajectory of the micropusher. The dynamic model proposed in this paper is able to determine the pusher trajectory in function of friction conditions. Based on this model, further works will be done to analyse the impact of the encapsulation on micropusher behavior. 
A new approach to manipulate a cell by using a small micropusher was described. The submerged ferromagnetic micropusher follows the movement of a magnet located in the air. We show that the manipulation of oocyte is possible with this device. A complete generic model of the micropusher trajectory is proposed. It enables to predict the micropusher movement in function of the magnet position according to the micropusher shape and properties and magnetic field induced by the magnet. This generic model can be generalized to other magnetic sources like micro-coils. This simulation tool will be the base of further works on the optimization of the micropusher architecture and on the development of automatic pushing tasks.

\section{ACKNOWLEDGMENTS}

This research was supported by the LAB (Laboratory of Automation of Besançon - France). The authors thank Prof. C. Roux (research group on "genetic and reproduction", Besançon) for collaboration on human oocytes micromanipulations. The authors thank the LCEP-Besançon for the fabrication of the micropusher glass sample support.

\section{REFERENCES}

[1] F. Arai, H. Maruyama, T. Sakami, A. Ichikawa, and T. Fukuda, "Pinpoint injection of microtools for minimally invasive micromanipulation of microbe by laser trap," IEEE/ASME Trans. on Mechatronics, vol. 8, no. 1, pp. 3-9, 2003.

[2] M. Frenea, S. P. Faure, B. L. Pioufle, P. Coquet, and H. Fujita, "Positioning living cells on a high-density electrode array by negative dielectrophoresis," Materials Science and Engineering, vol. 23, pp. 597-603, 2003.

[3] D.-H. Kim, B. Kim, S. Yun, and S. Know, "Cellular force measurement for force reflected biomanipulation," in Proceedings of the 2004 IEEE ICRA, New Orleans, USA, April 2004, pp. 2412-17.

[4] G. Li, N. Xi, M. Yu, F. Salem, D. H. Wang, and J. Li, "Manipulation of living cells by atomic force microscopy," in Proceedings of the 2003 IEEE/ASME International Conference on Advanced Intelligent Mechatronics (AIM 2003), Port Island, Kobe, Japan, July 2003, pp. 862-67.

[5] Y. Sun and B. J. Nelson, "Mems for cellular force measurements and molecular detection," Journal of Information Acquisition (IJIA), vol. 1, pp. 23-32, 2004.

[6] Y. Sun, K. T. Wan, B. J.Nelson, J. Bischof, and K. Roberts, "Mechanical property characterization of the mouse zona pellucida," IEEE Transactions on NanoBioScience, vol. 2, no. 4, pp. 279-286, 2003.

[7] M. Gauthier and E. Piat, "Control of a particular micro-macro positionning system applied to cell microdisplacement," IEEE Transactions on Automation Science and Engineering, vol. 3, no. 3, pp. 264-271, july 2006.

[8] —_ "An electromagnetic micromanipulation system for single cell manipulation," Journal of Micromechatronics, vol. 2, no. 2, pp. 87-119, Feb 2004.

[9] B. Bae, N. Kim, H. Kee, S.-H. Kim, Y. L., S. Lee, and K. Park, "Feasibility test of an electromagnetically driven valve actuator for glaucoma treatment," Journal of Microelectromechanical Systems, vol. 11, no. 4, pp. 344-54, august 2002.

[10] T. Bourouina, E. Lebrasseur, G. Reyne, A. Debray, H. Fujita, A. Ludwig, E. Quandt, H. Muro, T. Oki, and A. Asaoka, "Integration of two degree-of-freedom magnetostrictive actuation and piezoresistive detection: application to a twodimensional optical scanner," Journal of Microelectromechanical Systems, vol. 11, no. 4, pp. 355-61, august 2002.

[11] O. Cugat, J. Delamare, and G. Reyne, "Magnetic micro-actuators and systems (magmas)," IEEE Transactions on Magnetics, vol. 39, no. 6, pp. 3607-12, nov. 2003.

[12] H. Guckel, "Progress in magnetic microactuators," Microsystem technologies., vol. 5, no. 2, pp. 59-64, 1998.

[13] S. Guo, Y. Sasaki, and T. Fukuda, "A new kind of microrobot in pipe using driving fin," in Proceedings of the 2003 IEEE/ASME International Conference on Advanced Intelligent Mechatronics (AIM 2003), Port Island, Kobe, Japan, July 2003, pp. 697-702.

[14] A. Hatch, A. E. Kamholz, G. Holman, P. Yager, and K. F. Böhringer, "A ferrofluidic magnetic micropump," Journal of Microelectromechanical Systems, vol. 10, no. 2, pp. 215-221, June 2001.

[15] L. K. Lagorce, O. Brand, and M. Allen, "Magnetic microatuator based on polymer magnets," Journal of micromechanical systems, vol. 8, no. 1, pp. 3-14, 1999.

[16] C. Liu and Y. Y. W., "Micromachined magnetic actuators using electroplated permalloy," IEEE transactions on magnetics, vol. 35, no. 3, pp. 1976-85, 1999.

[17] J. Y. Park and M. G. Allen, "Development of magnetic materials and processing techniques applicable to integrated micromagnetic devices," Journal of Micromechanics and Microengineering, vol. 8, no. 4, pp. 307-316, 1998.

[18] K. B. Yesin, K. Vollmers, and B. J. Nelson, "Analysis and design of wireless magnetically guided microrobots in body fluids," in Proceedings of the 2004 IEEE ICRA, New Orleans, USA, April 2004, pp. 1333-38.

[19] F. Arai, H. Maruyama, T. S. aadn A. Ichikawa, and T. Fukuda, "Non-contact manipulation of biological cells," in Proceedings of the Workshop Microrobtics for biomanipulation, IROS03, Las Vegas, USA, Oct 2003, pp. 50-60. 
[20] M. Gauthier and E. Piat, "Microfabrication and scale effect studies for a magnetic micromanipulation system," in Proc. of the IEEE International Conference on Intelligent Robots and Systems - IROS02, vol. 2, Lausanne - Switzerland, 30 sept - 4 Oct 2002, pp. 1754-59.

[21] A. Ichikawa, F. Arai, H. Maruyama, T. Fukuda, and T. Katsuragi, "Single cell trap on a chip using in-situ microfabrication with photo-crosslinkable resin and thermal gelation," in Proceedings of the 2004 IEEE ICRA, New Orleans, USA, April 2004, pp. 2848-53. 